\title{
Using the SenseCam to Improve Classifications of Sedentary Behavior in Free-Living Settings
}

\author{
Jacqueline Kerr, PhD, Simon J. Marshall, PhD, Suneeta Godbole, MPH, \\ Jacqueline Chen, BS, Amanda Legge, BS, Aiden R. Doherty, PhD, Paul Kelly, MS, \\ Melody Oliver, PhD, Hannah M. Badland, PhD, Charlie Foster, PhD
}

\begin{abstract}
Background: Studies have shown relationships between important health outcomes and sedentary behavior, independent of physical activity. There are known errors in tools employed to assess sedentary behavior. Studies of accelerometers have been limited to laboratory environments.
\end{abstract}

Purpose: To assess a broad range of sedentary behaviors in free-living adults using accelerometers and a Microsoft SenseCam that can provide an objective observation of sedentary behaviors through first person-view images.

Methods: Participants were 40 university employees who wore a SenseCam and Actigraph accelerometer for 3-5 days. Images were coded for sitting and standing posture and 12 activity types. Data were merged and aggregated to a 60 -second epoch. Accelerometer counts per minute (cpm) of $<100$ were compared with coded behaviors. Sensitivity and specificity analyses were performed. Data were collected in June and July 2011 and analyzed in April 2012.

Results: TV viewing, other screen use, and administrative activities were correctly classified by the 100-cpm cutpoint. However, standing behaviors also fell under this threshold, and driving behaviors exceeded it. Multiple behaviors occurred simultaneously. A nearly 30-minute per day difference was found in sedentary behavior estimates based on the accelerometer versus the SenseCam.

Conclusions: Researchers should be aware of the strengths and weaknesses of the 100-cpm accelerometer cutpoint for identifying sedentary behavior. The SenseCam may be a useful tool in free-living conditions to better understand health behaviors such as sitting.

(Am J Prev Med 2013;44(3):290-296) @ 2013 American Journal of Preventive Medicine

\section{Introduction}

A number of studies have recently emerged that demonstrate a relationship between sedentary behavior and important health outcomes, independent of physical activity. ${ }^{1}$ Some employed questionnaires using a single measure of sedentary behavior: TV viewing. ${ }^{2-5}$ In some studies, participants reported overall

From the Department of Family and Preventive Medicine (Kerr, Marshall, Godbole, Chen, Legge), University of California San Diego, La Jolla, California; British Heart Foundation Health Promotion Research Group, Department of Public Health (Doherty, Kelly, Foster), University of Oxford, United Kingdom; Centre for Physical Activity and Nutrition (Oliver), Auckland University of Technology, New Zealand; and McCaughey VicHealth Centre for the Promotion of Mental Health and Community Wellbeing (Badland), University of Melbourne, Victoria, Australia

Address correspondence to: Jacqueline Kerr, PhD, Department of Family and Preventive Medicine, University of California San Diego, 9500 Gilman Drive 0811, La Jolla CA 92093-0811. E-mail: jkerr@ucsd.edu. $0749-3797 / \$ 36.00$

http://dx.doi.org/10.1016/j.amepre.2012.11.004 sitting time, ${ }^{6,7}$ or multiple sitting behaviors were summed. ${ }^{8}$ In others, accelerometers were employed and a cutpoint of less than 100 counts per minute (cpm) was used to represent either time in sedentary behavior ${ }^{9,10}$ or time spent without interruptions in sedentary behavior. ${ }^{11,12}$ Despite the inconsistency in measurement procedures, researchers and some government agencies conclude that reducing sedentary behavior may be a viable new health promotion strategy. ${ }^{13-15}$

There are known errors, however, in the tools employed to measure sedentary behavior. Self-report methods (e.g., questionnaires/surveys or diaries) are commonly used to assess sedentary behavior because they can identify specific behavior types, ${ }^{16-18}$ but self-report data are often plagued by memory recall errors, such as omission and telescoping, and other forms of bias associated with comprehension and positive representation. ${ }^{19} \mathrm{Be}$ cause of the error in self-reported data, researchers 


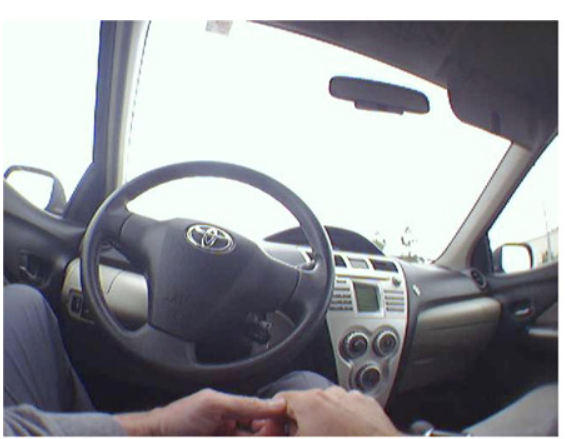

Sitting driving car

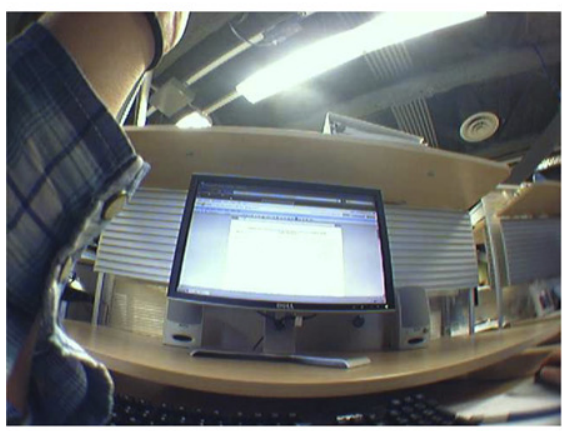

Sitting using computer screen

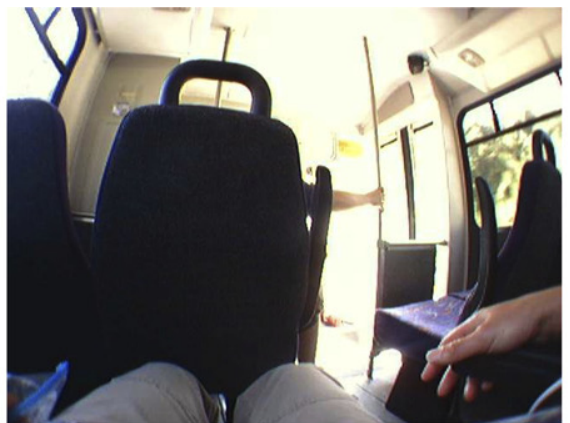

Sitting riding on a bus

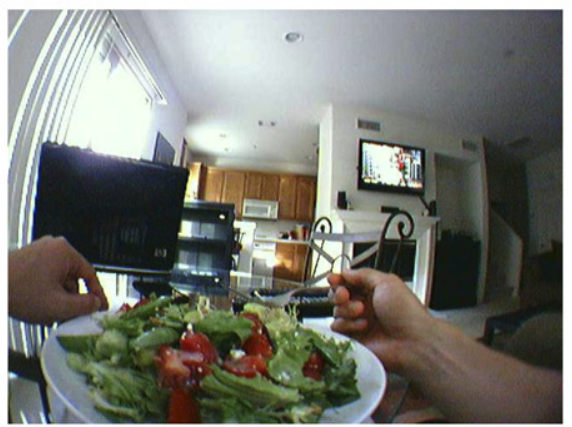

Sitting eating and watching TV

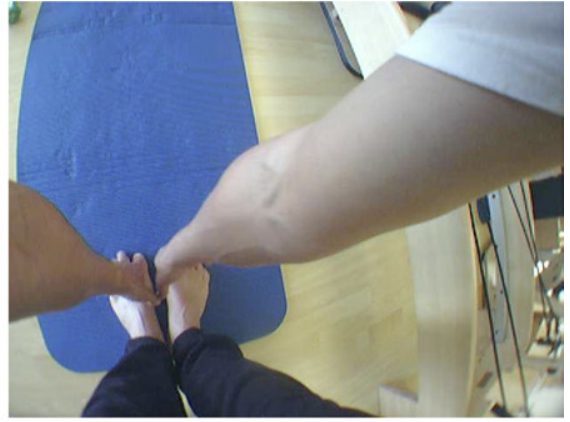

Standing doing conditioning exercise

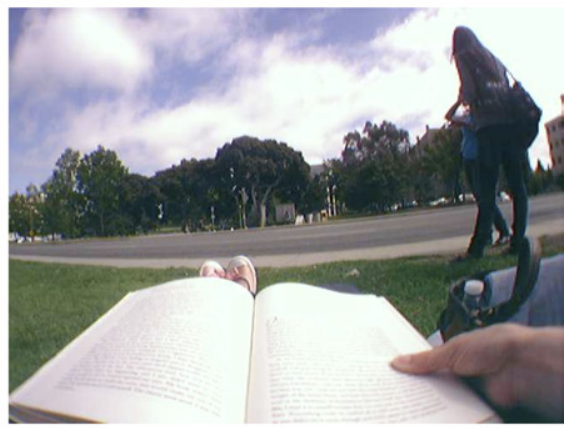

Sitting reading

Figure 1. Sample images of sedentary behavior from Microsoft's SenseCam

started using objective measures of sedentary behavior, employing hip-mounted accelerometers, a method that carries a relatively low participant burden and is feasible for use with large populations. ${ }^{20}$

Laboratory studies, however, have found that only $50 \%-60 \%$ of sedentary behavior is accurately classified using established cutpoints. ${ }^{21-26}$ In addition, sedentary behavior identified through accelerometry is also frequently indistinguishable from nonwear time. ${ }^{27,28} \mathrm{Al}$ though direct observation is the criterion standard for behavioral assessment, it is impractical in free-living settings. For this reason, accelerometer-based estimates of free-living sedentary behavior have scarcely been evaluated, and human observation periods in free-living studies have been short. ${ }^{29,30}$ Researchers may be mischaracterizing the prevalence of sedentary behavior, relationships between sedentary behavior and health, as well as the efficacy of interventions designed to reduce sedentary behavior.

A proxy for direct observation that is feasible to deploy in free-living environments is the Microsoft SenseCam, a device worn around the neck that automatically captures thousands of daily photographic images from the wearer's perspective. ${ }^{31}$ The SenseCam provides an important measurement advantage over other measures because it enables simultaneous domain and contextual information about sedentary behavior to be collected. Based on image data, inferences can be made about the type and context of sedentary behavior, which can be matched exactly to date-and-time-stamped estimates from an accelerometer. Therefore, the purpose of this paper is to present convergent validity evidence for accelerometerbased estimates of free-living sedentary behavior using contemporaneous photographic image data from a SenseCam.

\section{Methods}

\section{Participants}

Participants were adult cyclists recruited through a universitybased cycle-to-work network. Eligible participants were aged 18-70 years, were university employees, routinely bicycled for transportation, and were able to complete surveys in English. All study procedures were approved by the research ethics board of the University of California, San Diego. Commuting university employees who cycle were actively recruited because cycling is a behavior thought to be misclassified (as sedentary behavior) by accelerometers, and because many university employees have sedentary occupations. The fact that oversample cycling yielded a sufficient number of examples of it in the data set is notable.

Participants provided informed consent and agreed to wear the SenseCam devices and ActiGraph hip-worn accelerometer during waking hours for 3-5 days. A 3-5-day monitoring period (versus the conventional 7-day period for physical activity studies) was chosen because the goal was to garner a sufficient number of case examples rather than a stable estimate of habitual behavior. Approximately $50 \%$ of the sample wore the device during the weekend, and on at least 1 work day; the remainder wore the unit on weekdays only. At the completion of data collection, participants 
were given an opportunity to view and delete any images that they did not want included in the study (Figure 1 shows sample images). Data were collected in June and July 2011, and analyzed in April 2012.

\section{Measures}

Participants wore a GT3X+ ActiGraph accelerometer on a belt on their right hip that was timesynched with the SenseCam. Accelerometer data were processed using ActiLife 6.2.1 software, with 30-Hertz data aggregated to 60 seconds. Accelerometer-based sedentary behavior was computed as the number of minutes spent below $100 \mathrm{cpm}$ from vertical $(y)$-axis data, the most widely used cutpoint to determine sedentary behavior. ${ }^{20}$ Accelerometer nonwear time was defined as 90 minutes of consecutive zeros. ${ }^{26}$ All valid minutes were used in the analysis.

Details of the SenseCam system are described elsewhere ${ }^{31}$ and in this issue. ${ }^{32}$ Participants wore the SenseCam on a lanyard around their neck with adhesive clothing tape attached to reduce movement. The device takes photos every $10-15$ seconds, so the typical number of images taken per minute ranges from three to ten. Photos are taken when an onboard sensor is activated (e.g., by a change in movement, light, temperature, or presence of another person). If no photo is triggered by the sensors, a photo is taken every 50 seconds. More than 3000 wide-angle low-resolution images can be collected in 1 day.

Participants were required to charge the device each night and received daily reminder texts to comply with the protocol. Participants also were instructed on how to use a "privacy button" on the device, which prevented the device from capturing image data for up to 7 minutes. Participants were instructed to remove the SenseCam in locations where cameras were not permitted (e.g., fitness facilities), and to use the privacy button for activities such as bathroom visits and banking. Participants also were encouraged to ask for others' permission to record images during private or confidential meetings.

\section{SenseCam Image Coding}

SenseCam image data were downloaded and imported into the Clarity SenseCam Browser. ${ }^{33}$ A standardized coding protocol was developed based on existing behavioral taxonomies (e.g., SOFIT ${ }^{34}$ ) and refined using principles of nominal group technique. ${ }^{35}$ Interrater reliability of image coding was established using an iterative cycle of blind-coding (relative to other coders) followed by discussion, with all disagreements resolved by group consensus. This yielded a set of images with criterion codes attached from which additional coders could be trained and certified. Approximately $10 \%$ of all subsequent images were checked to minimize observer drift. Coders also received additional training in protecting the privacy, confidentiality, and security of the images. The full coding manual is available from the first author on request.
A series of at least five consecutive images (approximately 2 minutes) in the same behavior were grouped as an "event" and assigned a corresponding behavior code. First, "sedentary posture" was defined as sitting, lying, or reclining. A series of visual cues in each image were used to code the posture, including knee and leg positions, hands resting on a table, or camera angles that were lower than other people who were standing. Second, "standing" was defined as standing, moving in place, or moving toward an object. Visual cues to code "standing" included height and distance to furniture or other standing people, and absence of knees or legs in the image.

When objects in the image appeared in the same place from one image to the next, "standing without movement" was coded. If movement was observed, this was coded as "standing with movement." If progress toward a distant point was observed, this was coded as "walking/running." "Bicycling" was coded when handlebars were present in the image, and both street and stationary cycling were coded as such. Activities characterized as a transition from one posture to another were coded as "position transition."

After "posture" was coded, additional activity codes were assigned based on a published physical activity compendium. ${ }^{36}$ These codes included household activity, self-care, conditioning exercise, sports, manual labor, leisure, administrative activity, riding in car, riding in other vehicles, TV watching, other screen use, and eating. "Household activity" included behaviors such as cooking, washing dishes, and doing laundry. Actions coded as "selfcare" included brushing teeth, dressing, and putting on makeup. "Conditioning exercise" included such activities as weighttraining, yoga, and video workouts. Images of the wearer playing basketball, tennis, soccer, or other sports were coded as "sports." The "manual labor" code was assigned to activities such as shoveling, yard work, and carpentry. Pastimes such as painting and playing an instrument were categorized as "leisure."

"Administrative activity" included clerical duties such as writing, filing, and desk work. Transport modes such as the bus, train, or trolley were defined as "other vehicle" for any vehicle other than a car. The classification of "other screen use" was defined as the presence of any screen other than a TV. Eating was defined as the presence or observed consumption of food and beverages. In cases in which multiple behaviors were observed in a single image (e.g., eating and watching TV), the image was assigned both codes. Images were classified as "uncodeable" when the camera lens was obstructed. 
Table 2. Minutes of coded sedentary posture from Microsoft's SenseCam by activity category

\begin{tabular}{|lrccr|}
\hline Image code & Minutes & $\begin{array}{c}\text { Percent time } \\
\text { in } \\
\text { accelerometer } \\
\text { cpm }<100\end{array}$ & $\begin{array}{c}\text { Interquartile } \\
\text { range of } \\
\text { accelerometer } \\
\text { cpm }\end{array}$ & $\begin{array}{c}\text { Mean } \\
\text { accelerometer } \\
\text { cpm }\end{array}$ \\
\hline Sports & 0 & - & $1525-2900$ & 2330 \\
Self care & 85 & 60 & $2-361$ & 284 \\
Manual labor & 202 & 35 & $41-669$ & 432 \\
Conditioning exercise & 230 & 21 & $85-1405$ & 1262 \\
Household activity & 244 & 58 & $10-305$ & 260 \\
Riding in other vehicle & 409 & 82 & $0-50$ & 90 \\
Leisure & 428 & 81 & $0-73$ & 91 \\
Riding in car & 4,653 & 74 & $8-103$ & 101 \\
Eating & 5,250 & 92 & $0-4$ & 54 \\
TV watching & 5,407 & 89 & $0-11$ & 46 \\
Administrative activity & 9,546 & 92 & $0-10$ & 55 \\
Other screen use & 22,881 & 93 & $0-0$ & 34 \\
\hline
\end{tabular}

cpm, counts per minute

\section{Data Analysis}

SenseCam codes were aggregated to the minute level. A valid minute was defined as having the same posture and activity codes for the first and last image within the minute. SenseCam minutelevel and accelerometer minute-level data were merged using the time-stamp data for each unit. Time spent in sedentary behavior postures and activities as coded by the SenseCam were compared to the classification from the accelerometer. Minutes in each behavior, minutes under the $100-\mathrm{cpm}$ threshold, and mean counts in each behavior type were calculated. The sensitivity and specificity of the 100-cpm cutpoint was compared with the SenseCamderived classification of sedentary behavior, calculated for the whole data set and each participant. All analyses were performed using SPSS, version 19.

\section{Results}

A total of 40 participants completed the study. Each participant contributed a mean of 4 days of data; $70 \%$ were male; more than one third reported an annual household income of $\geq \$ 100,000$; average age was 36 years $(S D=12)$; and $85 \%$ were Caucasian. A total of 170 days were coded, including 86,109 valid minutes and 364,841 images. A total of 8546 minutes of images (8.2\%) were classified as "uncodeable," such as when the camera lens was obscured by an item of clothing or body part. It took trained coders approximately 2 hours to code 1 day of data including the six postures and 12 activity types. Participants were compliant with the wear instructions and did not report any problems with the devices. Several participants deleted a small number of images in the review process. Many participants were fascinated by the technology and research applications.

The coded image data were compared to the accelerometer data using the $100-\mathrm{cpm}$ threshold. On average, the accelerometer cutpoint classified participants in sedentary behavior for 331.2 minutes per day $(\mathrm{SD}=135.8)$. The average minutes coded as sedentary per day from the SenseCam images was $302.2(\mathrm{SD}=130.0)$. The majority of minutes were spent in a sitting posture (Table 1).

The accelerometer 100 cpm correctly identified "sitting" $90 \%$ of the time. However, when the SenseCam image indicated "standing without movement" or "standing with movement," the accelerometer recorded $<100 \mathrm{cpm} 72 \%$ and $35 \%$ of the time, respectively. Eleven percent of SenseCam bicycling had $<100 \mathrm{cpm}$ on the accelerometer. Overall, the sensitivity and specificity for the whole data set were $90 \%$ and $67 \%$, respectively. The mean sensitivity across participants was $89 \%(\mathrm{SD}=7 \%)$, and the mean specificity was $69 \%(\mathrm{SD}=11 \%)$.

Table 2 presents the various activities that occurred when the SenseCam images were coded as "sitting." The most-prevalent behavior categories, as determined by the number of minutes recorded by the SenseCam, were (1) other screen use; (2) administrative activity; (3) TV watching; (4) eating; and (5) riding in a car. TV viewing constituted $11 \%$ of the total sedentary time, compared with other screen use, which made up $46 \%$ of total observed sedentary time. The accelerometer cutpoint of $100 \mathrm{cpm}$ was accurate almost $90 \%$ of the time in classifying each of these four individual behaviors. At least $26 \%$ of the time observed in a car had accelerometer counts $>100 \mathrm{cpm}$.

\section{Discussion}

This study is the first to assess the convergent validity of a widely used accelerometer-based cutpoint for classifying sedentary behavior over several days of free-living behavior in adults using a discrete observation method. The findings suggest that using $100 \mathrm{cpm}$ from the vertical axis 
of an ActiGraph may both overestimate and underestimate some sedentary behaviors and should be used with caution. The difference in daily estimates, however, was only 30 minutes, which, although important cumulatively over time, is much less than the difference between self-report and accelerometer data. ${ }^{24}$ Although the validity of this cutpoint has been questioned in laboratory studies, the current study documents the generalizability of this finding in multiple days of free-living behavior. This finding is likely to be robust because existing behavioral taxonomies and standardized coding protocols to determine behaviors evident in SenseCam images were used.

The results suggest that for many sitting behaviors, such as non-TV screen time, administrative activity, eating, and watching TV, an accelerometer cutpoint of 100 $\mathrm{cpm}$ is accurate almost $90 \%$ of the time. Of note is the fact that classification accuracy appears to be better in the field than in the laboratory, where misclassification rates of up to $50 \%$ have been demonstrated. ${ }^{21-26}$ This may be due to a more stringent behavioral criterion used in the laboratory where actual direct observation is used rather than the SenseCam-based proxy employed in the field. Alternatively, accuracy could be higher in the field because of less sampling error-that is, a broader repertoire of behaviors can be sampled together with more examples of individual behaviors in a free-living study conducted across multiple days. Also interesting to note is that many of the accelerometer mean counts during the main sitting behaviors were around $50 \mathrm{cpm}$, rather than $100 \mathrm{cpm}$.

Researchers also need to be cautious when using the 100 -cpm cutpoint for detecting the time spent in a car; the 100 -cpm cutpoint misclassified this category $26 \%$ of the time. This is a common sedentary behavior that has been shown to have relationships with the built environment. ${ }^{37}$ Counts per minute for car riding ranged from 0 to 3347 , although the mean was $100 \mathrm{cpm}$. Further, the current findings suggest that the $100-\mathrm{cpm}$ cutpoint should not be employed in intervention studies that attempt to interrupt sitting time with standing breaks. ${ }^{38}$ Standing behaviors were classified under the $100-\mathrm{cpm}$ threshold $72 \%$ of the time. Other devices, such as thighmounted inclinometers, may be better suited to assessing the change from sitting to standing in intervention studies. $^{39,40}$

Although the accelerometer cutpoints appear to perform well for some activities, researchers have now started to question the technical, conceptual, and empirical basis of this approach. ${ }^{39}$ More-advanced methods have now been proposed, and pattern-recognition and machine-learning approaches seem to address many of the problems inherent to count-based approaches. ${ }^{40}$ The SenseCam is particularly useful in providing objective behavioral and contextual data to support the development of these models. For example, supervised machine learning operates when software learning programs are trained with behavior-annotated data. To date, many studies have employed laboratory protocols with a small range of behaviors and distinct breaks between behaviors to train the algorithm. In contrast, coded SenseCam data allow program training using free-living participant data, which can be applied to epidemiologic cohorts. The authors have started to use SenseCam data in this way by examining the behaviors of women at risk for breast cancer. ${ }^{41}$

Automated image-capture data also may provide further contextual information for understanding correlates of sedentary behavior. Many behaviors, such as eating, occurred while sitting and in the presence of other people, but this information is not commonly noted in self-report measures. In addition, hip-stationary behaviors were observed that might be classified as sedentary behavior by an accelerometer but clearly are not (e.g., some resistance and conditioning exercises). In addition, many behaviors occurred concurrently, for example sitting, eating, and watching TV. Although the primary behavior, "sitting," is likely to be related to health outcomes, this is likely to be confounded by co-occurring dietary behavior, particularly when the health outcome relates to energy balance (in the case of obesity). This confounding also underscores the complexity of measuring sedentary behavior and how participants may struggle to parse out their time use accurately on self-report surveys.

In this population, non-TV screen time was more prevalent than $\mathrm{TV}$ viewing, suggesting that $\mathrm{TV}$ time alone should not be used as a proxy for total screen time or sedentary behavior in general. These data are also consistent with findings among children and youth. ${ }^{42}$ However, the monitoring period used in the present study may not have not captured all TV viewing, as it was only 4 days and did not include weekends for all participants, when the amount of TV viewing may be higher.

\section{Limitations}

Limitations of this study include the small sample size and the relatively homogenous demographic characteristics of the sample. Manual coding of the images is also time-consuming and coding errors can occur. Further, the SenseCam as an observation tool for behavioral assessment has not been validated against in-person observations. Receiver operating characteristic curve analyses were not performed, as they would be required for each behavior type, and the goal was not to develop new accelerometer cutpoints. Future studies also could compare breaks in sitting time with both methods. Although not a feature of this analysis, SenseCam data can provide addi- 
tional information about social and physical contexts in which behaviors occur, which could greatly inform sedentary behavior intervention development.

\section{Conclusion}

The current study allowed a better understanding of strengths and limitations of using accelerometers to measure sedentary behavior in a free-living context. Researchers often use the terms sedentary behavior and sitting interchangeably, yet the coding of sitting posture in the current study showed that not all sitting behavior is inactive. It also was determined that a "standing" posture may often be misclassified as sedentary behavior if researchers employ a 100 -cpm accelerometer cutpoint. This is important if standing conveys a health-protective effect over sitting. ${ }^{43,44}$ Researchers should be aware of the strengths and weaknesses of the 100-cpm accelerometer cutpoint for identifying sedentary behavior. ${ }^{45}$ The SenseCam may be a useful tool in free-living conditions to better understand health behaviors such as sitting.

This work was supported by the British Heart Foundation under grant number 021/P\&C/core/2010/HPRG; the Irish Health Research Board under grant number MCPD/2010/12; and Microsoft Research, through its $\mathrm{PhD}$ Scholarship Programme.

No financial disclosures were reported by the authors of this paper.

\section{References}

1. Thorp AA, Owen N, Neuhaus M, Dunstan DW. Sedentary behaviors and subsequent health outcomes in adults: a systematic review of longitudinal studies, 1996-2011. Am J Prev Med 2011;41(2):207-15.

2. Dunstan DW, Barr EL, Healy GN, et al. Television viewing time and mortality: the Australian Diabetes, Obesity and Lifestyle Study (AusDiab). Circulation 2010;121(3):384-91.

3. Krishnan S, Rosenberg L, Palmer JR. Physical activity and television watching in relation to risk of type 2 diabetes: the Black Women's Health Study. Am J Epidemiol 2009;169(4):428-34.

4. Chang PC, Li TC, Wu MT, et al. Association between television viewing and the risk of metabolic syndrome in a community-based population. BMC Public Health 2008;8:193.

5. Aadahl M, Kjaer M, Jørgensen T. Influence of time spent on TV viewing and vigorous intensity physical activity on cardiovascular biomarkers. The Inter 99 study. Eur J Cardiovasc Prev Rehabil 2007;14(5): $660-5$.

6. Katzmarzyk PT, Church TS, Craig CL, Bouchard C. Sitting time and mortality from all causes, cardiovascular disease, and cancer. Med Sci Sports Exerc 2009;41(5):998-1005.

7. Gierach GL, Chang SC, Brinton LA, et al. Physical activity, sedentary behavior, and endometrial cancer risk in the NIH-AARP Diet and Health Study. Int J Cancer 2009;124(9):2139-47.

8. Shields M, Tremblay MS. Sedentary behaviour and obesity. Health Rep 2008;19(2):19-30.
9. Matthews CE, Chen KY, Freedson PS, et al. Amount of time spent in sedentary behaviors in the U.S., 2003-2004. Am J Epidemiol 2008; 167(7):875-81.

10. Healy GN, Wijndaele K, Dunstan DW, et al. Objectively measured sedentary time, physical activity, and metabolic risk: the Australian Diabetes, Obesity and Lifestyle Study (AusDiab). Diabetes Care 2008;31(2):369-71.

11. Healy GN, Dunstan DW, Salmon J, et al. Breaks in sedentary time: beneficial associations with metabolic risk. Diabetes Care 2008; 31(4):661-6.

12. Cooper AR, Sebire S, Montgomery AA, et al. Sedentary time, breaks in sedentary time and metabolic variables in people with newly diagnosed type 2 diabetes. Diabetologia 2012;55(3):589-99.

13. Dunstan DW, Owen N. New exercise prescription: don't just sit there: stand up and move more, more often. Arch Intern Med 2012;172(6):500-1.

14. Dunstan DW, Howard B, Healy GN, Owen N. Too much sitting-a health hazard. Diabetes Res Clin Pract 2012;97:368 -76.

15. Manns PJ, Dunstan DW, Owen N, Healy GN. Addressing the nonexercise part of the activity continuum: a more realistic and achievable approach to activity programming for adults with mobility disability? Phys Ther 2012;92(4):614-25.

16. Marshall AL, Miller YD, Burton NW, Brown WJ. Measuring total and domain-specific sitting: a study of reliability and validity. Med Sci Sports Exerc 2010;42(6):1094-102.

17. Rosenberg DE, Bull FC, Marshall AL, Sallis JF, Bauman AE. Assessment of sedentary behavior with the International Physical Activity Questionnaire. J Phys Act Health 2008; 5(S1):S30-S44.

18. Rosenberg DE, Norman GJ, Wagner N, et al. Reliability and validity of the Sedentary Behavior Questionnaire for adults. J Phys Act Health 2010;7(6):697-705.

19. Sallis JF, Saelens BE. Assessment of physical activity by self-report: status, limitations, and future directions. Res Q Exerc Sport 2000;71(2):1-14.

20. Matthews C, Chen K, Freedson P, et al. Amount of time spent in sedentary behaviors in the U.S., 2003-2004. Am J Epidemiol 2008; 167(7):875-81.

21. Dijkstra B, Kamsma Y, Zijlstra W. Detection of gait and postures using a miniaturised triaxial accelerometer-based system: accuracy in community-dwelling older adults. Age Ageing. 2010;39(2):259-62.

22. Marshall AL, Rachele JN, Marshall LAJ, Lai J, Jones LV. Sit versus stand: can sitting be accurately identified using MTI accelerometer data? Med Sci Sports Exerc 2010;42(5):475.

23. McMahon GC, Brychta RJ, Chen KY. Validation of the Actigraph (GT3X) inclinometer function. Med Sci Sports Exerc 2010;42(5):489.

24. Hart TL, Ainsworth BE, Tudor-Locke C. Objective and subjective measures of sedentary behavior and physical activity. Med Sci Sports Exerc 2011;43(3):449-56.

25. Oliver M, Schofield GM, Badland HM, Shepherd J. Utility of accelerometer thresholds for classifying sitting in office workers. Prev Med 2010;51(5):357-60.

26. Choi L, Liu Z, Matthews CE, Buchowski MS. Validation of accelerometer wear and nonwear time classification algorithm. Med Sci Sports Exerc 2011;43(2):357-64.

27. Oliver M, Badland HM, Schofield GM, Shepherd J. Identification of non-wear time and sedentary behavior using accelerometry. Res Q Exerc Sport 2011;82(4):779-83.

28. Winkler EA, Gardiner PA, Healy GN, et al. Distinguishing true sedentary from accelerometer non-wearing time: accuracy of two automated wear-time estimations. Med Sci Sports Exerc 2009;41(5):171.

29. Hart TL, McClain JJ, Tudor-Locke C. Controlled and free-living evaluation of objective measures of sedentary and active behaviors. J Phys Act Health 2011;8(6):848-57.

30. Kozey-Keadle S, Libertine A, Lyden K, Staudenmayer J, Freedson PS. Validation of wearable monitors for assessing sedentary behavior. Med Sci Sports Exerc 2011;43(8):1561-7. 
31. Hodges S, Williams L, Berry E, et al. SenseCam: a retrospective memory aid. In: Dourish P, Friday A, eds. Ubicomp 2006, LNCS 4206. 2006:177-93.

32. Doherty AR, Hodges SE, King AC, et al. Wearable cameras in health: the state of the art and future possibilities. Am J Prev Med 2013;44(3): $320-3$.

33. Doherty AR, Moulin CJ, Smeaton AF. Automatically assisting human memory: a SenseCam browser. Memory 2011;19(7):785-95.

34. McKenzie TL, Sallis JF, Nader PR. SOFIT: system for observing fitness instruction time. J Teaching Phys Ed 1991;11:195-205.

35. Potter M, Gordon S, Hamer P. The Nominal Group Technique: a useful consensus methodology in physiotherapy research. NZ J Physiother 2004;32(3):126-30.

36. Ainsworth BE, Haskell WL, Herrmann SD, et al. 2011 Compendium of physical activities: a second update of codes and MET values. Med Sci Sports Exerc 2011;43(8):1575-81.

37. Kozo J, Sallis JF, Conway TL, et al. Sedentary behaviors of adults in relation to neighborhood walkability and income. Health Psychol 2012;31(6):704-13.

38. Gardiner PA, Eakin EG, Healy GN, Owen N. Feasibility of reducing older adults' sedentary time. Am J Prev Med 2011;41(2):174-7.
39. Heil DP, Brage S, Rothney MP. Modeling physical activity outcomes from wearable monitors. Med Sci Sports Exerc 2012;44(1S1):S50 -S60.

40. Staudenmayer J, Zhu W, Catellier DJ. Statistical considerations in the analysis of accelerometry-based activity monitor data. Med Sci Sports Exerc 2012;44(1S1):S61-S67.

41. Patterson RE, Rock CL, Kerr J, et al. Metabolism and breast cancer risk: frontiers in research and practice. J Acad Nutr Diet 2012 Nov 2. pii: S2212-2672(12)01426-8. dx.doi.org/10.1016/j.jand.2012.08.015.

42. Biddle SJ, Gorely T, Marshall SJ. Is television viewing a suitable marker of sedentary behavior in young people? Ann Behav Med 2009; 38(2):147-53.

43. Hamilton MT, Hamilton DG, Zderic TW. Role of low energy expenditure and sitting in obesity, metabolic syndrome, type 2 diabetes, and cardiovascular disease. Diabetes 2007;56(11):2655-67.

44. Lynch BM. Sedentary behavior and cancer: a systematic review of the literature and proposed biological mechanisms. Cancer Epidemiol Biomarkers Prev 2010;19(11):2691-709.

45. Healy GN, Clark BK, Winkler EA, Gardiner PA, Brown WJ, Matthews CE. Measurement of adults' sedentary time in population-based studies. Am J Prev Med 2011;41(2):216-27. 